\title{
Significance of Hematological Scoring System (HSS) in Early Diagnosis of Neonatal Sepsis
}

\author{
Aparna Narasimha • M. L. Harendra Kumar
}

Received: 11 December 2009/Accepted: 10 December 2010/Published online: 1 January 2011

(C) Indian Society of Haematology \& Transfusion Medicine 2010

\begin{abstract}
Neonates are easily prone for bacterial infection. Diagnosis of neonatal septicemia may be difficult as the early signs of sepsis may be subtle and different at different gestational ages. The present study was undertaken to assess the significance of the hematological scoring system (HSS) for early detection of neonatal sepsis. About 50 peripheral blood smears of all newborns collected were analysed for neonatal sepsis using the HSS of Rodwell et al. (J Pediatr 112:761-767, 1988). Analysis in our study found that an abnormal immature to total neutrophil ratio (I:T) followed by an abnormal immature to mature neutrophil ratio (I:M) were the most sensitive indicators in identifying infants with sepsis. The HSS is a simple, quick, cost effective tool which can be used as a screening test for early diagnosis of neonatal sepsis.
\end{abstract}

Keywords Hematological scoring system · Neonatal sepsis · Peripheral blood smears · Infections

\section{Introduction}

Early diagnosis of neonatal septicemia is a vexing problem because of its nonspecific clinical picture [1]. Bacterial infection in the newborn still account for a considerable morbidity and mortality. This is because the newborn especially the premature are prone to serious infections by

A. Narasimha $\cdot$ M. L. Harendra Kumar

Department of Pathology, Sri Devaraj Urs Medical College,

Tamaka, Kolar, India

A. Narasimha $(\square)$

No. 22, "Moyenvilla", Moyenville Road, Langford Town,

Bangalore 565025, India

e-mail: aparna_patho@yahoo.com organisms and partly because the signs of these infections may be absent or minimal and hard to detect. Thus fatal septicemia may occur with little warning [2]. Hence the timely diagnosis of sepsis in neonates is critical as the illness can be rapidly progressive and in some instances fatal [3].

The current study was undertaken to assess the significance of the hematological scoring system (HSS) for early detection of neonatal sepsis.

\section{Materials and Methods}

The present study is a prospective analysis of the hematologic profiles of 50 neonates admitted in the neonatal care unit of our hospital. Infants were enrolled in the study if there were predisposing perinatal factors or if there was clinical suspicion of sepsis.

The study included three groups:

Group 1 -infants with sepsis with positive blood cultures. Group 2-infants with probable infection with strong clinical history but negative blood cultures. Group 3 -normal infants without any evidence of sepsis. The blood samples were collected in non-siliconized Vaccutainer tubes with tripotassium EDTA as an anticoagulant. Peripheral blood smears were be prepared within 1-2 h of venipuncture, stained with Leishman stain and examined under oil immersion light microscopy at a final magnification of $\times 1000$. The sepsis work up included blood and CSF culture and routine blood counts along with the hematologic score. Total leucocyte count was obtained using micros 60 autoanalyzer and corrected for nucleated red blood cells. Differential counts were performed on Leishman stained smears and about 200 cells were counted. 
Table 1 Hematological scoring system

\begin{tabular}{lll}
\hline Criteria & Abnormality & Score \\
\hline Total WBC count & $\leq 5,000 / \mu \mathrm{l}$ & 1 \\
& $\geq 25,000$ at birth & 1 \\
& $\geq 30,000-12-24 \mathrm{~h}$ & \\
& $\geq 21,000-$ Day 2 onwards & \\
& No mature PMN seen & 2 \\
Total PMN count & Increased/decreased & 1 \\
& Increased & 1 \\
Immature PMN count & Increased & 1 \\
I:T PMN ratio & $\geq 0.3$ & 1 \\
I:M PMN ratio & Toxic granules/cytoplasmic & 1 \\
Degenerative changes in & vacuoles & \\
PMN & $\leq 150,000 / \mu 1$ & 1 \\
Platelet count & &
\end{tabular}

The normal values are

Total PMN count-1800-5400

Immature PMN count-600

Immature: Total PMN ratio- 0.120

Immature: Mature PMN ratio- $\geq 0.3$

The peripheral blood smears of all newborns from birth up to 1 week were analysed for early diagnosis of neonatal sepsis using the hematological scoring system of Rodwell et al. [4]. The HSS assigns a score of one for each of the seven criteria found to be significantly associated with sepsis (Table 1) with one exception. An abnormal total count is assigned a score of 2 instead of 1 , if no mature polymorphs are seen on the peripheral smear to compensate for the low I:M ratio. Sensitivity, specificity, positive and negative predictive values were evaluated for each of the seven criteria of HSS.

Hematologic scoring system of Rodwell et al. [4] includes the following:

a) White blood cell and platelet count

b) White blood differential count

c) Nucleated red blood cell count (to correct WBC count)

d) Assessment of neutrophil morphology for degenerative changes

Immature neutrophils include promyelocyte, myelocyte (Fig. 1), metamyelocytes and, band form (Fig. 2). Degenerative changes in neutrophils include vacuolization (Fig. 3), toxic granulations (Fig. 4), and Dohle bodies.

Interpretation is shown in Table 2. Scores are: minimum score is 0 and maximum score is 8 .

\section{Statistical Analysis}

To test the statistical significance of three different groups, we employed the Kruskal Willis non-parametric test and a $P$ value $<0.05$ was considered as significant.

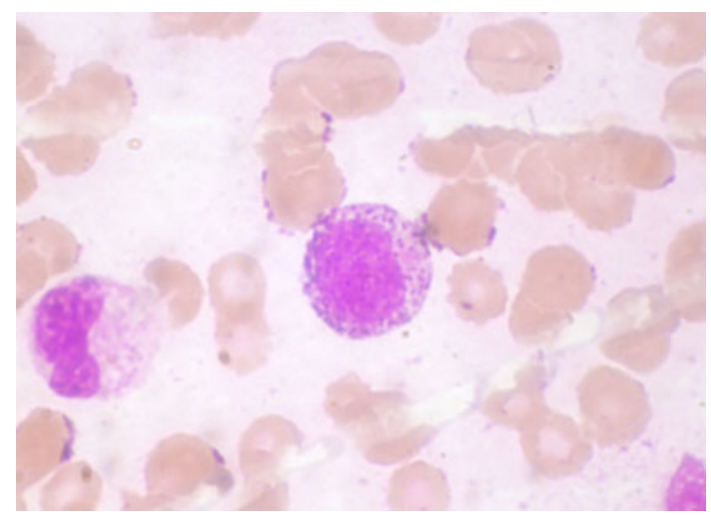

Fig. 1 Photomicrograph showing Immature neutrophils-Promyelocyte and myelocyte

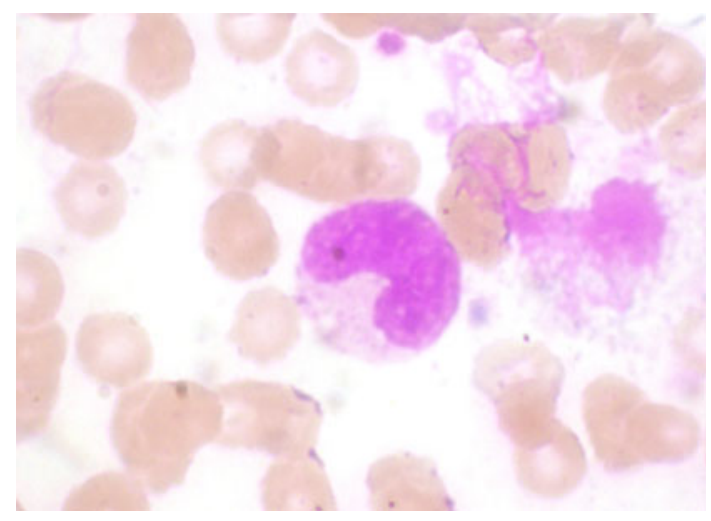

Fig. 2 Photomicrograph showing band form

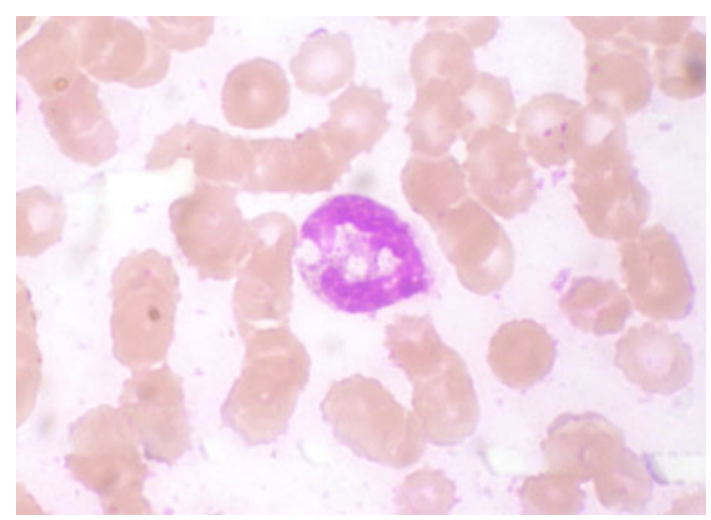

Fig. 3 Photomicrograph of neutrophil with cytoplasmic vacuoles

\section{Results}

The study included 50 cases divided into three groups represented in Table 3.

The diagnosis of sepsis was made when there were positive findings on blood culture. Infant were classified as having probable infection when the blood culture was negative but there was a strong clinical history for 


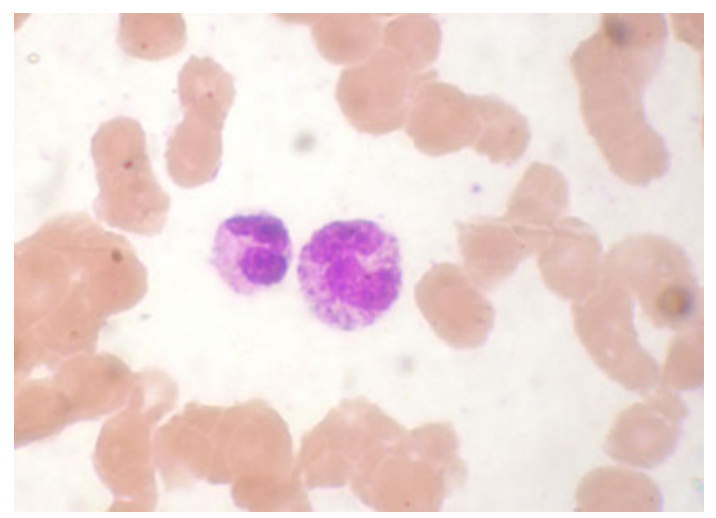

Fig. 4 Photomicrograph of neutrophils with toxic granules (Leishman, $\times 1000$ )

Table 2 Interpretation of hematological scoring system

\begin{tabular}{ll}
\hline Score & Interpretation \\
\hline$\leq 2$ & Sepsis is unlikely \\
3 or 4 & Sepsis is possible \\
$\geq 5$ & Sepsis or infection is very likely \\
\hline
\end{tabular}

Table 3 Group distribution of cases

\begin{tabular}{ll}
\hline Groups & Number of cases (\%) \\
\hline Group 1-sepsis & $12(24)$ \\
Group 2-probable infection & $26(52)$ \\
Group 3-Normal infants & $12(24)$ \\
\hline
\end{tabular}

Table 4 Age and sex distribution of cases

\begin{tabular}{lcc}
\hline Age & Males (\%) & Females (\%) \\
\hline $0-24$ h (D1) & $18(36)$ & $16(32)$ \\
$24-48$ h (D2) & $1(2)$ & $1(2)$ \\
$48-72$ h (D3) & $3(6)$ & $4(8)$ \\
$72-96$ h (D4) & - & $3(6)$ \\
$>96$ h & $3(6)$ & $1(2)$ \\
Total & $25(50)$ & $25(50)$ \\
\hline
\end{tabular}

infection. Infants were considered to be normal when the blood culture was negative and there was no strong clinical evidence of infection.

The study had equal distribution of males (50\%) as well as females (50\%) (Table 4). We had 29 (58\%) term infants and $21(42 \%)$ pre-term infants with the age ranging from $24 \mathrm{~h}$ to 8 days.

Table 5 shows scores of each groups: $12(100 \%)$ infants with history of sepsis had scores $\geq 5.11$ (42.30\%) infants with history of probable infection had scores $>5$ and 8
Table 5 Scores of each of the groups

\begin{tabular}{llll}
\hline Groups & $\begin{array}{l}\text { Score 0-2 } \\
(\%)\end{array}$ & $\begin{array}{l}\text { Score 3-4 } \\
(\%)\end{array}$ & $\begin{array}{l}\text { Score } \geq 5 \\
(\%)\end{array}$ \\
\hline $\begin{array}{l}\text { Sepsis (12 cases) } \\
\begin{array}{l}\text { Probable infection (26 } \\
\text { cases) }\end{array}\end{array}$ & $7(27)$ & $8(30.76)$ & $11(42.30)$ \\
\begin{tabular}{l} 
Normal (12 cases) \\
\hline
\end{tabular} & $5(41.66)$ & $5(41.66)$ & $2(16.66)$ \\
\hline
\end{tabular}

Note: Kruskal-Willis test $\left(\mathrm{x}^{2}-20.12, \mathrm{df}-2, P\right.$ value $\left.<0.005\right)$

$(30.76 \%)$ had scores $\geq 3-4$ suggesting the possibility of sepsis. $2(16.66 \%)$ of normal infants had score $>5$ suggesting the presence of sepsis and $5(41.66 \%)$ had scores $>3$ or 4 suggesting the possibility of sepsis in these cases. 5 $(41.66 \%)$ of normal infants and $7(27 \%)$ infants with probable infection had score $\leq 2$, which implies sepsis was unlikely in these cases.

Table 6 shows performance of individual hematologic findings: Total PMN count $(89.47 \%)$ was highly sensitive followed by Immature PMN count $(78.94 \%)$ in identifying infants with sepsis. Total leucocyte count (TLC) $(91.66 \%)$ followed by Immature: Total PMN ratio (75\%) and platelet counts $(75 \%)$ were highly specific tests helpful in diagnosing sepsis. The positive predictive value was high for Immature: Total PMN ratio $(88.88 \%)$ followed by platelet count $(85.71 \%)$ which was helpful in identifying infants who really had sepsis. Negative predictive value was high in degenerative changes $(40 \%)$ along with I:T PMN ratio which indicated that the infants did not have any evidence of sepsis.

\section{Discussion}

Neonatal sepsis, sepsis neonatorum, and neonatal septicemia are terms that have been used to describe the systemic response to infection in newborn infants. Sepsis in newborn can be a devastating problem leading to morbidity and mortality. The inability of neonates to completely muster the minimum inflammatory response makes them more susceptible to bacterial invasion of the blood stream than older children and adults and the risks are even higher in preterm infants. Diagnosis of neonatal septicemia may be difficult as the early signs of sepsis may be subtle and different at different gestational ages [5].

Inability to adequately exclude the diagnosis of neonatal sepsis can result in unnecessary and prolonged exposure to antibiotics. Thus laboratory tests that assist the clinician in diagnosis of infection in neonates have considerable relevance [6].

In our study considering all four parameters i.e: sensitivity, specificity, positive predictive value and negative predictive value, I:T PMN ratio and degenerative changes 
Table 6 Performance of individual hematologic findings

\begin{tabular}{lllll}
\hline & Sensitivity (\%) & Specificity $(\%)$ & $\begin{array}{l}\text { Positive predictive } \\
\text { value (PPV) }(\%)\end{array}$ & $\begin{array}{l}\text { Negative predictive } \\
\text { value (NPV) }(\%)\end{array}$ \\
\hline Total WBC count & 10.52 & 91.66 & 80 & 24.44 \\
Total PMN count & 89.47 & 8.33 & 75.55 & 20 \\
I PMN count & 78.94 & 8.3 & 73.17 & 11.11 \\
I:T PMN ratio & 63.15 & 75 & 88.88 & 39.13 \\
I:M PMN ratio & 73.68 & 50 & 82.35 & 37.5 \\
Degenerative changes & 68.42 & 66.66 & 66.66 & 40 \\
Platelet count & 47.36 & 75 & 85.71 & 31 \\
\hline
\end{tabular}

were the most reliable tests for diagnosing sepsis. An abnormal I:M PMN ratio was highly sensitive in identifying sepsis. Degenerative changes in neutrophils were not found to be a very sensitive indicator of sepsis. Thrombocytopenia was consistently associated with poor prognosis. These findings were in comparison with other studies $[3,4,7,8]$. The higher the score, the greater was the likelihood of sepsis. A score $\leq 2$ suggests that sepsis was unlikely.

Hematologic scoring system (HSS) can improve the diagnostic accuracy of complete blood count. It can be employed as a screening test for diagnosing sepsis. But it is important to simplify and standardize the interpretation of this global test [4].

Though there are several methods for rapid detection of microorganisms in blood cultures of newborn infants using automated blood culture system, DNA probe and fluorometric detection systems [9-11], still HSS can be employed as a useful test to distinguish the infected from the non infected infants. It has high sensitivity and specificity, the certainty of sepsis being present with higher scores.

\section{Conclusion}

HSS is a simple, quick, cost effective tool which can be used as a screening test for early diagnosis of neonatal sepsis. It may aid the clinicians in identifying sepsis and to institute proper anti-biotic therapy. Unnecessary exposure of infants to antibiotic therapy can thus be avoided.

Acknowledgments Mrs. Christina (Lab Technician) for technical help and Sri Devaraj Urs Academy of Higher Education and Research for funding.

\section{References}

1. Mathur NB, Saxena LM, Sarkar R, Puri RK (1994) Superiority of acridine orange-stained buffy coat smears for diagnosis of partially neonatal septicaemia. Acta Paediatr 83(6):652-655

2. Xanthour M (1970) Leucocyte blood picture in healthy full term and premature babies during neonatal period. Arch Dis Child 45:242-249

3. Speer CP, Gahr M, Schrotter W (1985) Early diagnosis of neonatal infection. Monatsschr Kinderheilkd 133(9):665-668

4. Rodwell RL, Leslie AL, Tudehope DI (1988) Early diagnosis of neonatal sepsis using a hematologic scoring system. J Pediatr 112:761-767

5. Neonatal bacteraemia diagnosis and management (editorial) (1997) Br Med J 2:1385-1386

6. Ghosh S, Mittal M, Jaganathan G (2001) Early diagnosis of neonatal sepsis using a hematological scoring system. Indian $\mathbf{J}$ Med Sci 55(9):495-500

7. Philip AGS, Hewitt JR (1980) Early diagnosis of neonatal sepsis. Pediatrics 65:1036-1041

8. Basu S, Guruprasad, Narang A, Garewal G (1999) Diagnosis of sepsis in the high risk neonate using a hematologic scoring system. Indian J Hematol Blood Transf 17:32-34

9. Gars-Prats JA, Cooper TR, Schneider VF, Stager CE, Hansen TN (2000) Rapid detection of microorganisms in blood cultures of newborn infants utilising an automated blood culture system. Pediatrics 105:523-527

10. Hertz D, Fuller D, Davis T, Popile L, Stevenson D, Lemons J (1999) Comparison of DNA probe technology and automated continuous monitoring blood culture systems in the detection of neonatal bacteraermia. J Perinatol 19:290-293

11. Pauli IJ, Lekhawat P, Kehl S, Sasidharan P (1999) Early detection of bacteraemia in the neonatal intensive care unit using the new BALTEC system. J Perinatol 19:127-131 